In press with The Astrophysical Journal

Preprint typeset using $\mathrm{LAT}_{\mathrm{E}} \mathrm{X}$ style emulateapj v. 5/2/11

\title{
A PERFECT STARBURST CLUSTER MADE IN ONE GO: THE NGC 3603 YOUNG CLUSTER
}

\author{
SAmbaran BanerJee and Pavel Kroupa \\ Argelander-Institut für Astronomie, Auf dem Hügel 71, D-53121, Bonn, Germany; \\ sambaran@astro.uni-bonn.de, pavel@astro.uni-bonn.de \\ In press with The Astrophysical Journal
}

\begin{abstract}
Understanding how distinct, near-spherical gas-free clusters of very young, massive stars shape out of vast, complex clouds of molecular hydrogen is one of the biggest challenges in astrophysics. A popular thought dictates that a single gas cloud fragments into many new-born stars which, in turn, energize and rapidly expel the residual gas to form a gas-free cluster. This study demonstrates that the above classical paradigm remarkably reproduces the well-observed central, young cluster (HD 97950) of the Galactic NGC 3603 star-forming region, in particular, its shape, internal motion and the mass distribution of stars, naturally and consistently follow from a single model calculation. Remarkably, the same parameters (star formation efficiency, gas expulsion time scale and delay) reproduce HD 97950 as were found to reproduce the Orion Nebula Cluster, Pleiades and R136. The present results thereby provide intriguing evidences of formation of star clusters through single-starburst events followed by significant residual gas expulsion.

Subject headings: stars: kinematics and dynamics - methods: numerical —open clusters and associations: individual(NGC 3603 young cluster) —galaxies: star formation-galaxies: starburst-galaxies: star clusters: general
\end{abstract}

\section{INTRODUCTION}

Very young, massive star clusters (hereafter VYMCs), which are compact associations of stars of mass $\gtrsim 10^{4} M_{\odot}$ and up to a few Myr old ${ }^{1}$, are typically found in locations of high star-formation activity (or "starburst regions") in our Milky Way and external galaxies. The classical notion implies that such systems form through a single episode of starburst. In this scenario, efficient cooling processes within a dense parent gas-cloud lead to the formation of a number of proto-stellar cores. Such an infant star cluster consists of massive main sequence (MS) and lower-mass pre-main-sequence (PMS) stars embedded within the residual gas. The radiation and winds from the MS and PMS stars inject energy into this gas until the latter becomes unbound and escapes the system. The associated rapid dilution of the potential well causes the system to expand which loses a fraction of its stars depending on its initial mass and concentration (Adams 2000; Kroupa et al. 2001; Boily \& Kroupa 2002; Baumgardt \& Kroupa 2007; Banerjee \& Kroupa 2013; Pfalzner \& Kaczmarek 2013). The remaining gasfree system may eventually attain a state of virial equilibrium. Such a "monolithic" formation scenario has successfully explained the structure and kinematics of Galactic and extra-galactic VYMCs like the Orion Nebula Cluster (ONC) and R136 and intermediate-aged clusters like the Pleiades and Hyades (Kroupa et al. 2001; Banerjee \& Kroupa 2013).

In this study we incorporate the same properties and parameters (see below) of the gas expulsion process as those in the earlier studies (Kroupa et al. 2001; Banerjee \& Kroupa 2013) in our realistically detailed model

\footnotetext{
${ }^{1}$ Such clusters are usually referred to as "starburst clusters" but here we prefer a more generic and origin-independent designation of such systems. They constitute a sub-category of Young Massive Star Clusters (Portegies Zwart et al. 2010).
}

calculations of the time-evolution of star clusters. We find good and consistent agreement between these model computations and detailed measurements of the central young cluster (HD 97950; hereafter HD97950) of the Galactic starburst region NGC 3603 which are based on infrared and optical observations with the ESO/VLT (Very Large Telescope) and the Hubble Space Telescope (HST), respectively (Harayama et al. 2008; Rochau et al. 2010; Pang et al. 2013). In particular, we closely reproduce its observed (a) mass density profile (Harayama et al. 2008), (b) central velocity dispersion (Rochau et al. 2010; Pang et al. 2013), (c) present-day stellar mass function (PDMF; Pang et al. 2013), and as well (d) the spatial distribution of stars from the crude data (Pang et al. 2013), simultaneously from one individual model calculation. This not only strongly points to a single-episode formation of HD97950 as envisaged previously (Stolte et al. 2004, 2006) but it also affirms the monolithic formation channel for VYMCs in general and suggests that such systems evolve according to universal principles.

\section{MODEL CALCULATIONS}

\subsection{Initial systems and gas dispersal}

We perform time $(t)$-evolutionary calculations that mimic gas-dispersal from model star clusters beginning from their gas-embedded phase. The star clusters are initialized by distributing stars over a highly compact (see below) and spherically-symmetric Plummer phasespace distribution (Heggie \& Hut 2003; Kroupa 2008) of total stellar mass $M_{e c l}$. The stellar initial mass function (IMF) follows the canonical two-part power-law (Kroupa 2001; Kroupa et al. 2013). The dominant physical effect of the embedding gas is taken into account by applying an external potential from a spherically-symmetric mass distribution of total mass $M_{g}$ following the same Plummer distribution as the stars. The gas-depletion is implemented by asymptotically reducing $M_{g}$ with a timescale 

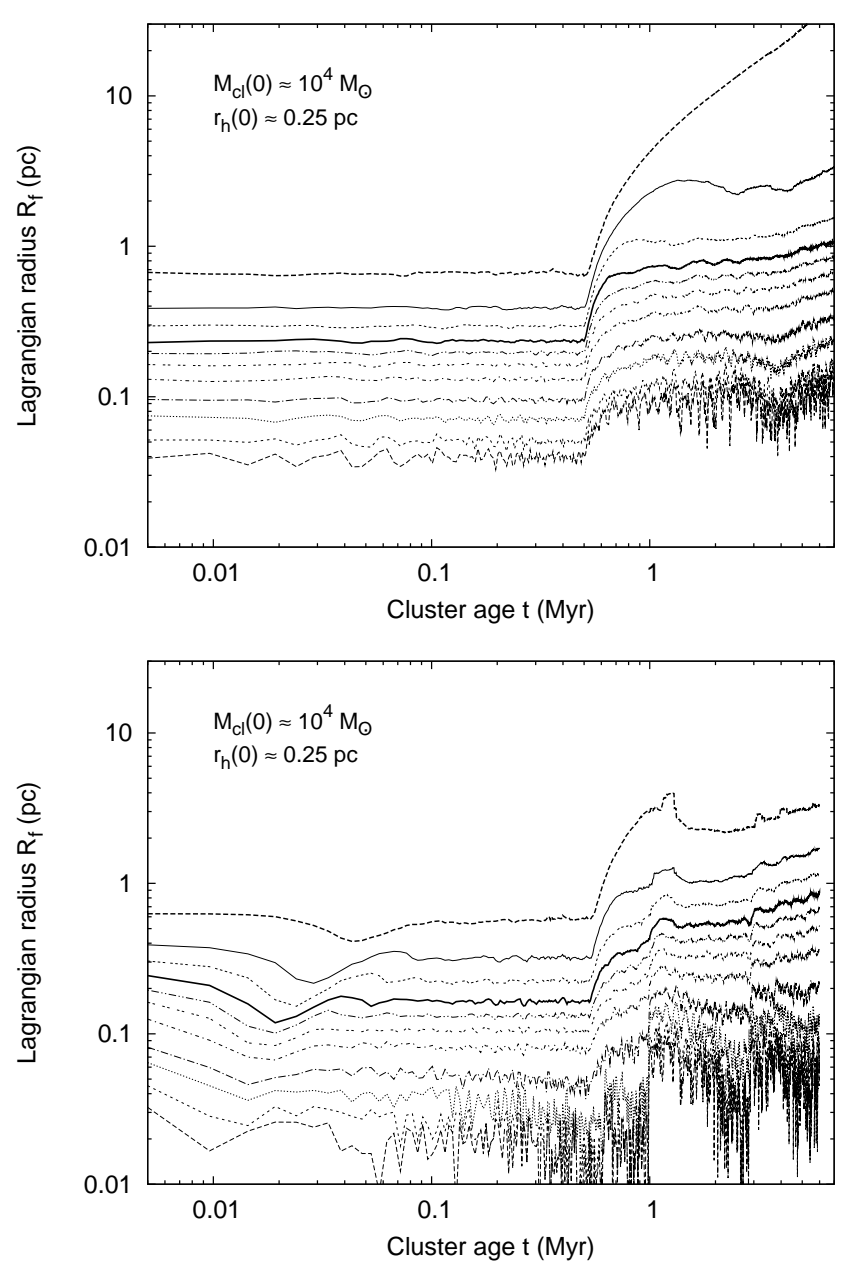

Figure 1. Lagrange radii $R_{f}$ for mass fraction $f$ for the model cluster computations without (top; model HD97950s in Table 1) and with (bottom; model HD97950b in Table 1) primordial binaries (Sec. 2). In each panel, the curves, from bottom to top, correspond to $f=0.01,0.02,0.05,0.1,0.2,0.3,0.4,0.5,0.625,0.7,0.9$ respectively. The Lagrange radii evolution shows a rapid expansion of the clusters at $t_{d} \approx 0.6 \mathrm{Myr}$ due to gas expulsion followed by re-collapse of a fraction of them to dynamical equilibrium. For the computation with binaries (Sec. 2.1.2), the cluster undergoes a brief initial collapse phase or "binary cooling" (Sec. 3.1). In the top panel (no primordial binaries), the $R_{0.9}$ blows up due to the inclusion of escapers.

$\tau_{g}$ after an embedded-phase time-span of $\tau_{d}$ as in Kroupa et al. (2001); Banerjee \& Kroupa (2013). Detailed Herschel observations indeed indicate Plummer-like sections (Malinen et al. 2012) of highly compact (about $0.1 \mathrm{pc}$ in section) gas filaments within which (and at their intersections) the spherical/spheroidal proto clusters form via gravitational fragmentation and collapse (Schneider et al. 2010, 2012; Hill et al. 2011; Hennemann et al. 2012). Adopting an appropriately time-varying background potential, mimicking the effect of the gas, is currently the only viable method to computationally deal with the complex magneto-hydrodynamic plus radiation transfer problem on this mass scale (see Sec. 4).

\subsubsection{Gas dispersal}

We adopt the same values of the above parameters quantifying the overall gas expulsion as in Kroupa et al. (2001) and Banerjee \& Kroupa (2013). Both ob- servations (Lada \& Lada 2003) and theoretical studies (Machida \& Matsumoto 2012) support a local starformation efficiency (SFE) of $\epsilon \approx 1 / 3$, i.e., $M_{g}(0)=$ $2 M_{\text {ecl }}(0)$ initially $(t=0$; see more in Sec. 4$)$.

The timescales $\tau_{d}$ and $\tau_{g}$ depend on the complicated physics of gas-radiation interaction. For simplicity, we estimate $\tau_{g}$ by taking the average radial speed of the expelling gas to be the sound speed in ionized hydrogen (i.e., that of an HII region), viz., $v_{g} \approx 10 \mathrm{~km} \mathrm{~s}^{-1}$. This sound speed corresponds to the typical temperature of $\approx 10^{4} \mathrm{~K}$ of an HII region (Osterbrock 1965; Anderson et al. 2009 $)^{2}$. In other words, $\tau_{g}=r_{h}(0) / v_{g}$, where $r_{h}(0)$ is the initial half-mass radius $(\approx$ virial radius for a Plummer model) of the stellar cluster/gas. The residual gas begins to launch when it becomes ionized by the (proto)stellar radiation so that the latter is coupled efficiently with the gas. The coupling of the radiation with the resulting HII medium substantially overpressures the latter and can even make it radiation-pressure-dominated (RPD) which might briefly drive the gas supersonically in the beginning (Krumholz \& Matzner 2009). The expanding gas continues to move out with the sound speed of an HII region once it becomes gas-pressure-dominated (GPD) (Hills 1980). This initial RPD phase is essential for launching the gas from massive clusters whose escape speed exceeds the HII sound speed. Therefore, for massive clusters, the above $\tau_{g}$, from $v_{g} \approx 10 \mathrm{~km} \mathrm{~s}^{-1}$, gives an upper limit (also see Kroupa 2005); it can be shorter depending on the duration of the RPD state (also see Sec. 4).

This $\tau_{g}$ is typically comparable to the dynamical time (or crossing time over half-mass radius) $\tau_{\text {cr }}$ of the embedded cluster. This makes the gas expulsion process "explosive" (Kroupa 2005), where the cluster is unable to adjust itself to the varying gas potential and hence expands "violently". It can be shown (Kroupa 2005) that for moderate to massive clusters, the energy (wind+radiation) injected in the gas by the OB stars within a dynamical time is sufficient to make the gas unbound, supporting an explosive nebula disruption.

As for the delay-time, we take $\tau_{d} \approx 0.6$ Myr as a representative value (Kroupa et al. 2001). The value of $\tau_{d}$ again depends on gas-radiation interaction. An estimate of $\tau_{d}$ can be obtained from the lifetimes of the Ultra Compact HII (UCHII) regions which can be upto $\approx 10^{5} \mathrm{yr}(0.1 \mathrm{Myr}$; Churchwell 2002). The highly compact pre-gas-expulsion clusters (Sec. 3 ) have sizes $\left(r_{h}(0)\right)$ only a factor of $\approx 3-4$ larger than the typical size of a UCHII region $(\approx 0.1 \mathrm{pc})$. If one applies a Strömgren sphere expansion scenario (Churchwell 2002 and references therein) to the compact embedded cluster, the estimated delay-time, $\tau_{d}$, before a sphere of radius $r_{h}(0)$ becomes ionized, would also be larger by a similar factor and hence close to the above representative value. Once the gas gets ionized, it couples efficiently with the radiation (pressure) from the $\mathrm{O} / \mathrm{B}$ stars and is launched

${ }^{2} T \approx 10^{4} \mathrm{~K}$ is the thermal equilibrium temperature where the heating by the ionizing UV radiation (primarily from the OB stars) is compensated by the cooling due to the free electrons (mostly via free-free and free-bound transitions) in the ionized medium. Neutral hydrogen (HI) regions, on the contrary, are much colder $(<100 \mathrm{~K})$. The effective ionization in HII (by UV) causes efficient coupling of the radiation flux with the free electrons and hence results in much more efficient energy transfer than that in HI. 
Table 1

Initial and gas expulsion parameters for the computed models that reproduce well observed VYMCs (very young massive clusters; see Sec. 1). The models HD97950s/b refer to the ones with initially single-only stars/primordial binaries as computed here (see Sec. 3). Also included are the previously published model clusters that agree well with the VYMCs R136 (Banerjee \& Kroupa 2013) and ONC (and also Pleiades; Kroupa et al. 2001).

\begin{tabular}{lccccccccc}
\hline \hline Model cluster & $M_{\mathrm{ecl}}(0) / M_{\odot}$ & $M_{g}(0) / M_{\odot}$ & $r_{h}(0) / \mathrm{pc}$ & $\tau_{g} / \mathrm{Myr}$ & $\tau_{\mathrm{cr}}(0) / \mathrm{Myr}$ & $\tau_{d} / \mathrm{Myr}$ & $f_{b}(0)$ & $Z / Z_{\odot}$ & Reference \\
\hline HD97950s & $1.0 \times 10^{4}$ & $2.0 \times 10^{4}$ & 0.25 & 0.025 & 0.029 & 0.6 & 0.0 & 1.0 & this paper \\
HD97950b & $1.0 \times 10^{4}$ & $2.0 \times 10^{4}$ & 0.25 & 0.025 & 0.025 & 0.6 & 1.0 & 1.0 & this paper \\
\hline R136 & $1.0 \times 10^{5}$ & $2.0 \times 10^{5}$ & 0.45 & 0.045 & 0.021 & 0.6 & 0.0 & 0.5 & Banerjee \& Kroupa (2013) \\
ONC-B & $4.2 \times 10^{3}$ & $8.4 \times 10^{3}$ & 0.21 & 0.021 & 0.066 & 0.6 & 1.0 & 1.0 & Kroupa et al. (20001) \\
\hline
\end{tabular}

Note. - The initial gas mass $M_{g}(0)$ and the gas expulsion timescales $\tau_{g}$ and $\tau_{d}$ (see Sec. 2.1.1) are consistent with $\epsilon \approx 0.33$, $v_{g} \approx 10 \mathrm{~km} \mathrm{~s}^{-1}\left(\tau_{g}=r_{h}(0) / v_{g}\right)$ and $\tau_{d} \approx 0.6 \mathrm{Myr}$ for all these computed models. See Sec. 4 for further discussions.

immediately (see above). High-velocity outflows from proto-stars (Patel et al. 2005) additionally aid the gas expulsion. A more elaborate calculation of the expansion of a Strömgren front through the proto-cluster gas is underway (Pflamm-Altenburg, in preparation).

Admittedly, the above arguments ignore complications such as unusual morphologies of UCHIIs and possibly non-spherical ionization front, among others, and only provide basic estimates of the gas-removal timescales. Observationally, Galactic $\approx 1$ Myr old gas-deprived VYMCs such as the ONC and the HD97950 imply that the embedded phase can be $\tau_{d}<1$ Myr. The above widely-used analytic gas-expulsion model does realize the essential dynamical effects on the star cluster after the residual gas is depleted. Moreover, the above same values of $\epsilon \approx 0.33, \tau_{d} \approx 0.6 \mathrm{Myr}$ and $\tau_{g}=r_{h}(0) / v_{g}\left(v_{g} \approx\right.$ $10 \mathrm{~km} \mathrm{~s}^{-1}$ ) that reproduced the ONC and the Pleiades (Kroupa et al. 2001) as well as the R136 (Banerjee \& Kroupa 2013) also fair excellently with the HD97950 as we shall see in the following sections.

\subsubsection{Primordial binaries}

Apart from initial systems consisting of only single stars as above, we also create initial systems with primordial binaries which is the realistic case. Here we use a $f_{b}(0)=100 \%$ primordial binary fraction at $t=0$ (Kroupa 1995a). For stars of mass $m<5 M_{\odot}$, the orbital period $(P)$ distribution of such binaries is given by the "birth period distribution" (Kroupa 1995a) which spans over a wide range of period between $1.0<\log _{10} P<8.43$ where $P$ is in days (Kroupa 1995b). We take a thermal distribution of binary eccentricities. With the dynamical evolution and eventual disruption (by the Galactic tidal field) of the parent cluster, such primordial binary population gives rise to the appropriate period distribution observed for low-mass stellar binaries in the solar neighborhood (Kroupa 1995a; Marks \& Kroupa 2011).

For massive stars of $m>5 M_{\odot}$, we adopt a much tighter and narrower initial period distribution given by a (bi-modal) Öpik law (uniform distribution in $\log _{10} P$ ) that spans over the range $0.3<\log _{10} P<3.5$ (Sana \& Evans 2011). This is motivated by the observed period distribution of O-star binaries in nearby O-star rich clusters (Sana \& Evans 2011; Chini et al. 2012). It is currently unclear at which stellar mass the orbital period law changes and how it changes and the above discontinuous switching of the $P$-distribution at $m=5 M_{\odot}$, therefore, had to be chosen somewhat arbitrarily. The consistency of the resulting break in the stellar mass function of the computed clusters with the observed one (see Sec. 3.1) provides a justification to the above switching. Apart from the MF-break, our results, as discussed below, are largely insensitive to the switching point of the period law (in the range $2 M_{\odot}-7 M_{\odot}$, see below).

For $m>5 M_{\odot}$, we furthermore use biased/ordered pairing in which the stars, picked by optimally sampling (Kroupa et al. 2013) the canonical IMF, are arranged in order of $m$ and paired sequentially. This is motivated by the observation that O-stars are typically found in binaries with O/B stars (Sana \& Evans 2011; Chini et al. 2012). For lower $m$, the stars are paired by picking each component randomly and independently from the canonical IMF. This is consistent with all available data on young and old Galactic field systems (Marks \& Kroupa 2011). Finally, for very low-mass stellar binaries with short $\left(P<10^{3} \mathrm{~d}\right)$ periods, the orbits are adjusted to account for PMS "eigenevolution" (Kroupa 1995b). This takes into account the very early (age $<10^{5} \mathrm{yr}$ ) system-internal orbital energy and orbital angular momentum redistribution due to, e.g., tidal circularization and system-internal residual gas.

\subsection{N-body computations}

We trace the time evolution of such initial systems (set using MCLUSTER; Küpper et al. 2011) using the state-ofthe-art direct N-body integration code NBODY6 (Aarseth 2003). Apart from utilizing a highly sophisticated numerical integration scheme, NBODY6 also keeps track of the evolution of the individual stars (Hurley et al. 2000). We use the iron metallicity of $Z=Z_{\odot}$ in all the calculations, as appropriate for HD97950. We explore a range of plausible initial clusters to spot one that reproduces the observed structure and kinematics of HD97950, being consistent with its age and photometrically-determined mass ranges of $1-2 \mathrm{Myr}$ and $10000 M_{\odot}-16000 M_{\odot}$, respectively (Stolte et al. 2004, 2006).

\section{RESULTS: A SURPRISINGLY WELL-MATCHING MODEL}

As expected, all computed clusters undergo a nearly steady, compact embedded state until the gas expulsion at $\tau_{d} \approx 0.6 \mathrm{Myr}$ from which they begin to expand (see Fig. 1, top panel). The cluster profile correspondingly changes from a highly concentrated and relatively static one to an expanded and rapidly changing profile. Fig. 2 (top, solid line and filled squares) shows the surface/projected mass density profile $\Sigma_{M}$ at $t \approx 1.2 \mathrm{Myr}$ for a computed cluster of only single stars with half- 

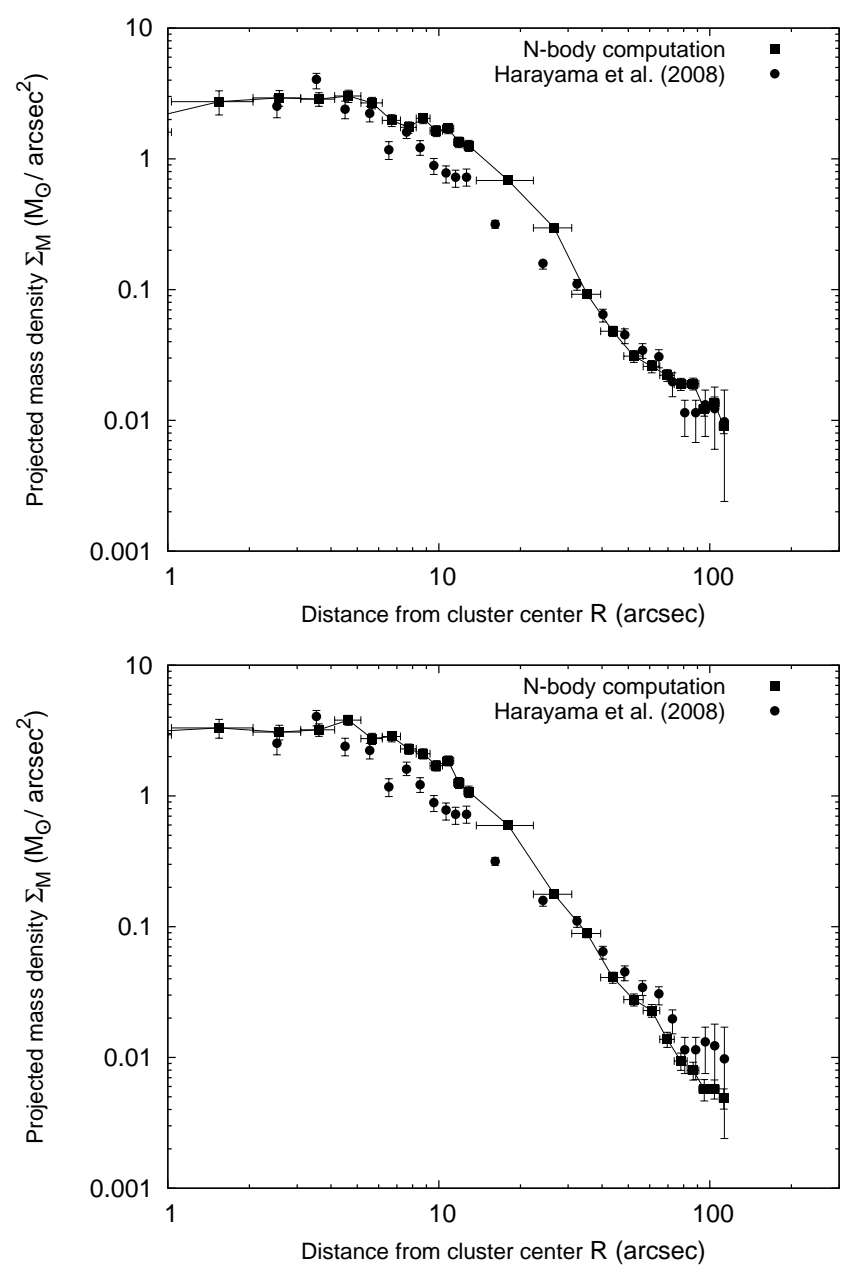

Figure 2. Top: The computed projected mass density profile (filled squares and solid line) for the stellar mass range $0.5 M_{\odot}<$ $m<2.5 M_{\odot}$ at $t \approx 1.2 \mathrm{Myr}$ from a model computation containing only single stars initially $\left(r_{h}(0) \approx 0.25 \mathrm{pc}, M_{\text {ecl }}(0) \approx 10000 M_{\odot}\right.$; same model HD97950s as shown in the top panel of Fig. 1). This profile shows remarkable agreement with the observed profile (Harayama et al. 2008), for the same stellar mass range, of the central young cluster (HD97950) of the NGC 3603 starburst region (filled circles). The angular annuli used for computing the projected densities (the horizontal error-bars) are nearly the same as those used to obtain the observed profile. Bottom: The computed profile of a star cluster having the same mass and size as above initially but with all stars in primordial binaries, showing excellent agreement with the observed profile at $t \approx 1.4 \mathrm{Myr}$. All legends are the same as above. This is the same model HD97950b as shown in the bottom panel of Fig. 1. Here, and for all the next figures, 1 arcsec $\approx 0.03 \mathrm{pc}$ as appropriate for HD97950.

mass radius $r_{h}(0) \approx 0.25 \mathrm{pc}$ and $M_{e c l}(0) \approx 10000 M_{\odot}$ for the stellar component at $t=0$ as shown for the model "HD97950s" in Table. 1 (hence $r_{h}(0) \approx 0.25 \mathrm{pc}$ and $M_{g}(0) \approx 20000 M_{\odot}$ for the gas, $\tau_{g} \approx 0.025$ Myr which is close to the initial dynamical time $\left.\tau_{\mathrm{cr}}(0) \approx 0.029 \mathrm{Myr}\right)$. It agrees remarkably with that observed in HD97950 (filled circles; Harayama et al. 2008). Note that in this comparison a similar stellar mass range and annuli as those for the observed profile are used to construct the density profile from the computed cluster.

How well does this matching model reproduce other observed properties of HD97950? An important check is to compare with the cluster's central stellar velocity dispersion which is obtained through proper motion mea- surements (Rochau et al. 2010; Pang et al. 2013). The above computed model, however, gives a rather lower (1-dimensional) velocity dispersions $\left(\sigma_{1 d} \approx 3 \mathrm{~km} \mathrm{~s}^{-1}\right)$ for $1<t<2$ Myr than what is observed in HD97950, viz., $\sigma_{1 d}=4.5 \pm 0.5 \mathrm{~km} \mathrm{~s}^{-1}$ for stars of masses between $1.7 M_{\odot}<m<9.0 M_{\odot}$ within $R<15^{\prime \prime}(\approx 0.5 \mathrm{pc})$ from the cluster center (Rochau et al. 2010). This is shown in Fig. 3 (top).

\subsection{Computations with primordial binaries}

Interestingly, this deficiency is compensated if one instead considers the computed models with primordial binaries (Sec. 2.1.2). Fig. 3 (middle) shows the $\sigma_{1 d}$ evolution for a computed model with $f_{b}(0)=100 \%$ primordial binary fraction but otherwise identical to the above single-star model at $t=0$ (model "HD97950b" in Table 1 ), for the same zone and mass range as above. The augmented values of $\sigma_{1 d}$ (of the COMs, i.e., excluding the binaries' internal orbital motion) in this case can be attributed to frequent binary-single and binary-binary encounters that release the internal binding energy of the binaries ("binary-heating"; Heggie \& Hut 2003). Here, the values of $\sigma_{1 d}$ lie between $4.5-7.0 \mathrm{~km} \mathrm{~s}^{-1}$ which includes the observed value. Notably, the models with primordial binaries undergo an initial contraction by $\approx 10 \%$ as shown in Fig. 1 (bottom), unlike the singlestar models. This is caused by the kinetic energy absorbed in ionizing loosely-bound or "soft" binaries which are present in large numbers initially (Kroupa 1995a; Sec. 2.1.2). This effect can therefore be referred to as "binary-cooling" (Kroupa et al. 1999). In fact, the presence of a large number of soft binaries effectively cause a moderately sub-virial initial system which collapses and virializes (after re-expanding; c.f., Fig. 1) in a few crossing times. The presence of a gas potential deepens this initial collapse. Note that this effect is a direct result of the chosen initial binary $P$-distribution (c.f., Sec. 2.1.2). This distribution is successful in reproducing the observed $P$-distribution of low-mass stellar field binaries in the solar neighborhood (Kroupa 1995a,b; Marks \& Kroupa 2012) and hence constitutes a realistic initial condition. The details of this process will be studied in a future paper.

However, the corresponding initial collapse does not conflict with the intended $\epsilon \approx 0.33$ as this SFE corresponds to that of the overall system, i.e., the total starto-gas ratio of the whole initial cluster. This is what determines the post-gas-expulsion violent relaxation (expansion) of the cluster. This initial shrinkage would cause the cluster to attain the observed density profile somewhat later in time (see below).

A recent work (Banerjee et al., in preparation), in which an HD79750-like massive cluster forms via merging of much less-massive sub-clusters within a gas potential, shows that a similarly compact cluster as in the present case is formed right before gas expulsion. The formation of such a massive cluster is, of course, true only if the implied gas density is very high.

The above computed model with binaries as well reproduces the observed projected mass density profile at $t \approx 1.4$ Myr, shown in Fig. 2 (bottom). Fig. 4 shows the profile of the incompleteness-limited stellar number density $\Sigma_{N}$ from the above computed cluster (filled squares 

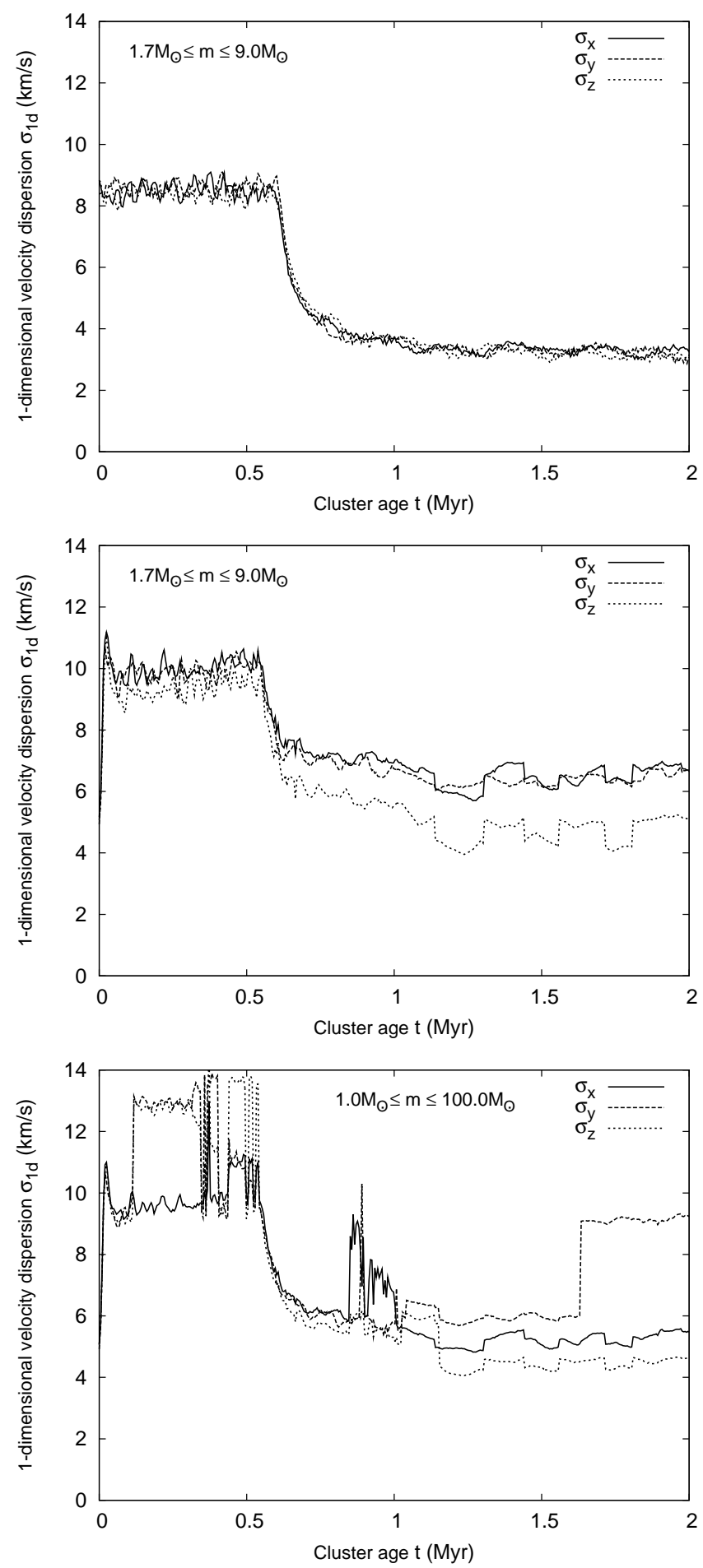

Figure 3. The time evolution of the 1-dimensional velocity dispersions, $\sigma_{1 d}$, for the model cluster computations without (top) and with (middle) primordial binaries (see text), the density profiles of which are shown in Fig. 2. They are obtained for $R<0.5 \mathrm{pc}$ $\left(\approx 15^{\prime \prime}\right)$ and $1.7 M_{\odot}<m<9.0 M_{\odot}$ consistently with the observations of Rochau et al. (2010). The bottom panel shows the $\sigma_{1 d}$ evolution in the latter computation but for $1.0 M_{\odot}<m<100.0 M_{\odot}$ as in Pang et al. (2013). For the binaries in the computed model, the center-of-mass velocities, giving their overall motion through the cluster, has been used while their most massive/brightest member stars (primaries) refer to the masses. The computed values of $\sigma_{1 d}$ (bottom panel) differ in orthogonal directions as found in observations (Pang et al. 2013) and span the same range as observed (4.5-7.0 $\mathrm{km} \mathrm{s}^{-1}$ ) between 1.0 - 1.5 Myr cluster age, implying good agreement. and solid line) at $t \approx 1.4 \mathrm{Myr}$ and that obtained from HST (Pang et al. 2013) (filled triangles) which are in excellent agreement. In this comparison, we have sampled the computed distribution according to the radius and mass-dependent incompleteness fraction particular to this observation (Pang et al. 2013), to mimic the "observation" of the model cluster. In constructing both of these density profiles we include only the most massive member (primary) of a binary which would dominate the detected light from it.

Fig. 3 (bottom) shows the $\sigma_{1 d}$ evolution in the binary model over $1.0 M_{\odot}<m<100.0 M_{\odot}$ within $R<0.5$ pc. The computed dispersion of the velocity components lie between $4.0<\sigma_{1 d}<7.0 \mathrm{~km} \mathrm{~s}^{-1}$ for $1<t<2 \mathrm{Myr}$. The corresponding measured 1-d velocity dispersions do change substantially with orthogonal directions (Pang et al. 2013) like the computed ones (see Fig. 3; bottom panel) and their variations closely agree to the above computed range. The abrupt vertical excursions in $\sigma_{1 d}$ in Fig. 3 (bottom) originate from energetic two- or fewbody encounters which are most frequent for the most massive stars and binaries as they centrally segregate the most via two-body relaxation.

Finally, Fig. 5 (bottom) shows the computed global $\left(R<60^{\prime \prime}\right.$ or $\left.\approx 1.8 \mathrm{pc}\right)$ stellar mass function (again, of only the primaries) or the present day mass function (PDMF) at $t \approx 1.4 \mathrm{Myr}$ (filled squares). The PDMF shows a vivid break at $\log \left(m / M_{\odot}\right) \approx 0.7$ beyond which it becomes much shallower, with slope $\Gamma=-0.92 \pm 0.13$, than the canonical IMF. This $\Gamma$ is in marked agreement with the observed $\Gamma=-0.88 \pm 0.15$ (for $0.6<$ $\log \left(m / M_{\odot}\right)<2.0$ ) over the same projected area (Pang et al. 2013). Downwards the break, $\Gamma \approx-1.35$, the canonical value. This break, which has been anticipated by Kroupa et al. (2013), can be attributed to the switching of the binary period distribution to much tighter binaries for the massive stars $\left(m>5 M_{\odot}\right.$; see Sec. 2.1.2). The massive-stellar binaries being much more tightly bound than the low-mass ones, the massive stars retain a much higher binary fraction with time than the low-mass stars. Therefore, upwards the MF break, one excludes a good fraction of lower-mass secondaries while detecting only the primaries, thereby effectively flattening the PDMF, unlike downwards $\left(m<5 M_{\odot}\right)$. Fig. 5 (top and middle) demonstrates the development of this break as an increasing number of low-mass binaries ionize.

Interestingly, the corresponding observed PDMF shows a similar break (Pang et al. 2013) which is significant but less vivid and is at somewhat lower $m$. In reality, it can be expected that such a break in the PDMF would be moderately different than that obtained here. This is because the change in the binary period distribution law would be more continuous than what is assumed here. Note that Pang et al. (2013) also obtained their (observed) $\Gamma$ upwards this break like the computed one here.

\section{DISCUSSION AND CONCLUSION}

This study demonstrates that it is possible to reproduce the detailed observed features of the central cluster HD97950 of the NGC 3603 starburst region, within its age and mass constraints, from a monolithic formation standpoint and adopting the reasonable and widely used values of the parameters quantifying the gas dispersal 


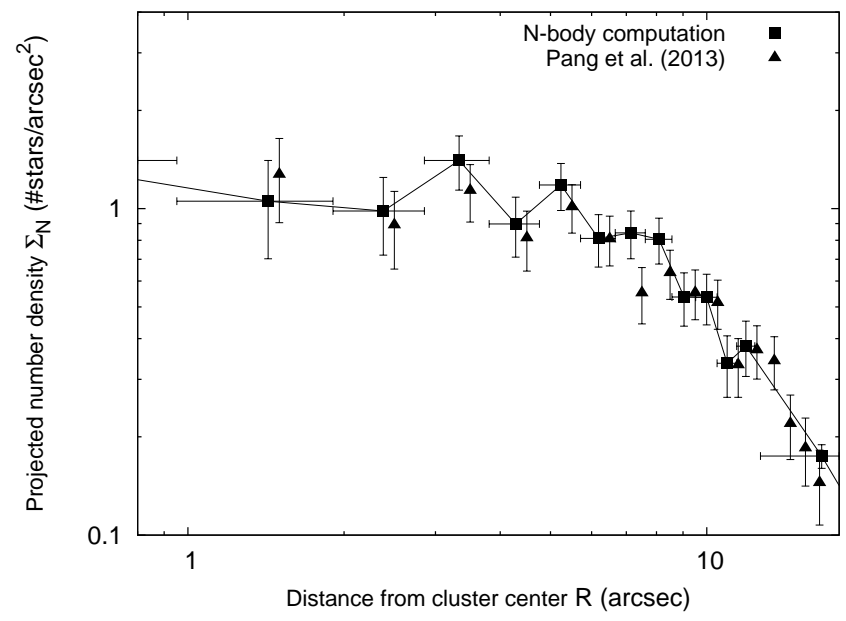

Figure 4. The computed projected stellar number density profile for the calculation HD97950b (Table 1) with primordial binaries (filled squares and solid line; c.f. Fig. 2, text). This shows a remarkable agreement at $t \approx 1.4 \mathrm{Myr}$ with the same obtained with the stars of HD97950 from the HST/PC chip (extends up to central 15" ; filled triangles; Pang et al. 2013). In constructing the computed profile, we take into account the incompleteness in the detection of the stars that depends on the stellar mass (luminosity) and projected angular annuli on the cluster (Pang et al. 2013). In this comparison, similar angular annuli (horizontal error-bars) have been used to construct the density profiles.

phase (Kroupa et al. 2001; Banerjee \& Kroupa 2013). It should be noted that the above results imply a natural match of the computed models with the observed data without any scaling or fitting.

The above calculations have been performed without applying an external Galactic potential. As verified by comparing with calculations with a Solar-neighborhoodlike field, the effect of the external field is only minor. This can be expected as the highly compact initial system remains well within its Roche lobe. Notably, the initial compact size $\left(r_{h}(0) \approx 0.25 \mathrm{pc}\right)$ of the computed models is consistent with the observed widths of the compact gas-filaments that host the proto-clusters (André et al. 2011) and also with the mass-radius relation of embedded clusters (Marks \& Kroupa 2012).

In this work, we assume the "classical" SFE of $\epsilon \approx$ $33 \%$ over the proto-cluster scale which is supported by both theoretical (Machida \& Matsumoto 2012) and observational (Lada \& Lada 2003) studies. We do not take into account the variations of SFE over the protostar/subcluster scale as suggested in recent theoretical studies (Girichidis et al. 2012; Moeckel et al. 2012). Such studies, based on hydrodynamic simulations, indicate much higher local SFE which would influence the gas expulsion. However, inclusion of proto-stellar outflows have been shown to substantially suppress the local SFE to $\lesssim 50 \%$ and is consistent with $\approx 33 \%$ SFE over both local (stellar) and global (proto-cluster) scale (Price \& Bate 2010; Machida \& Matsumoto 2012).

The analytic potential-lowering method applied here is widely used to this date for a good reason. The self-consistent treatment of gas removal requires threedimensional radiation-hydrodynamic calculations which is formidable to date for the mass scale involved in this study. High-resolution (reaching the "opacity-limit") smoothed-particle-hydrodynamics (SPH) computations have been done so far forming stars in gas-spheres of only up to $\approx 500 M_{\odot}$ (Klessen et al. 1998; Bate \& Bonnell 2004; Bate 2009; Girichidis et al. 2011, 2012; Bate 2012) but without any feedback and hence self-regulation mechanism. Radiation-magnetohydrodynamic (MHD) calculations incorporating feedback to the star-forming gas has also been carried out from proto-stellar scales (Machida \& Matsumoto 2012; Bate et al. 2013) upto $\approx 50 M_{\odot}$ gas spheres (Price \& Bate 2010). While the latter studies provide insights into the self-regulation mechanisms in the star formation process, the processes that lead to the ultimate dispersal of the gas is still unclear. Therefore, the analytic potential lowering is the best that can be done now for the mass scales involved here.

It should be noted, however, that the above monolithic cluster formation scenario has nevertheless successfully reproduced ONC, Pleiades and Hyades clusters previously (Kroupa et al. 2001) and as well the R136 (Banerjee \& Kroupa 2013) and the HD97950 cluster as in here. This indicates that although deprived of the details of star-formation and radiation-hydrodynamic processes due to technological bottleneck, the adapted simplified gas-expulsion model and the choices of the parameters might still be an appropriate description of the overall gas expulsion process. Indeed, in a comparative study, Geyer \& Burkert (2001) found that analytic gaspotential reduction has essentially the same effect on the cluster evolution as in an SPH computation (for a given SFE) where the gas is removed via shock heating. The present work thereby provides another pillar to the todate widely used classical gas expulsion scenario.

As such, the current state-of-the-art in theoretical research on star formation does not provide a definitive answer to the gas dispersal and cluster formation processes and their interrelations. Given this, the present work provides a plausible set of physical conditions that compellingly evolve to reproduce the detailed observed properties of HD97950, subject to its photometric and age constraints. They comprise a solution since the same computed model reproduces multiple observed properties of the cluster (its density profiles, velocity dispersion and stellar mass function). Remarkably, the same conditions $\left(\epsilon \approx 0.33, v_{g} \approx 10 \mathrm{~km} \mathrm{~s}^{-1}, \tau_{g}=r_{h}(0) / v_{g}\right.$, $\left.\tau_{d} \approx 0.6 \mathrm{Myr}\right)$ that reproduced the known properties of the ONC and Pleiades (Kroupa et al. 2001) also works for the much more massive R136 and HD97950 clusters (see Table 1). The present work thereby serves as an intriguing evidence that these clusters might have formed monolithically with the chosen parameters being an appropriate description of the overall gas expulsion process.

Several authors alternatively propose VYMCs to be formed via hierarchical merging of subclusters/structures (Fujii et al. 2012; Smith et al. 2013; Longmore et al. 2014). In particular, Fujii et al. (2012) have demonstrated agreements with observed radial cumulative distributions of massive stars and merger products in HD97950. This work, although interesting, cannot be taken as a vivid counterexample because of notable drawbacks. Here, the representative age of HD97950 has been taken to be 2.5 Myr which is far too old compared to the best-fitting age of $\approx 1$ Myr with only a small age spread, as obtained from the cluster's PMS-MS CMD (Stolte et al. 2004; Pang et 
al. 2013). In $1 \mathrm{Myr}$, the substructures hardly merge to form a single cluster (c.f., Fig. 2 of Fujii et al. 2012). Furthermore, no attempt has been made to compare directly measurable quantities like the structure and kinematics of the merged cluster with observations. Hence, it still remains to be seen how well a hierarchical merging scenario can reproduce the structure and kinematics of HD97950 given its very young age. Based on the current theoretical literature, it is as such impossible to rule out a single-starburst scenario of the formation of smooth-structured VYMCs in preference to a hierarchical one, on fundamental grounds.

Notably, the construction of spatial density profiles involves accumulation over finite-sized annuli and the heights over the profile depends on the choice of these annuli. However, note that in Fig. 2, the angular annuli are chosen nearly the same as those used by Harayama et al. (2008) to obtain their observed profile. Also, in Fig. 4, the same angular annuli are used to construct both of the number density profiles.

Several earlier authors have considered HD97950 to be in complete virial equilibrium and estimated its dynamical mass to be $\approx 20000 M_{\odot}$ from its observed velocity dispersion (Rochau et al. 2010; Pang et al. 2013). We have computed initially $M_{e c l}(0) \approx 20000 M_{\odot} \bmod -$ els (with $\epsilon \approx 33 \%$ ) to find that such massive systems yield either too centrally-dense or too expanded profiles, i.e., no well-matching initial conditions could be found. The $M_{e c l}(0) \approx 10000 M_{\odot}$ models presented here, that do reproduce the observations, are not in dynamical equilibrium at the epoch of matching $(t \approx 1.4 \mathrm{Myr})$, but are still expanding (the innermost regions are close to re-collapsing). A prediction from this study, therefore, is that the stars in the outer parts of HD97950 should have outgoing radial proper motions which can be tested by future observations.

Fig. 6 shows radial profiles of $\sigma_{1 \mathrm{~d}}$ at $t=1.4 \mathrm{Myr}$ for $1.7 M_{\odot} \leq m \leq 9.0 M_{\odot}$ and $1.0 M_{\odot} \leq m \leq 100.0 M_{\odot}$ where $\sigma_{1 \mathrm{~d}}$ tends to increase for $R \gtrsim 40^{\prime \prime}(\approx 1.2 \mathrm{pc})$. The latter feature can be attributed to the recent gas expulsion from the system resulting in its outer parts still expanding. Such a trend, which becomes more pronounced the closer the epoch of observation is to the gas expulsion, can be tested by future, more accurate determinations of stellar proper motions in the outer regions of HD97950, e.g., by Gaia. Notably, the measured tangential velocities of selected stars in Pang et al. (2013) do show an increasing trend with radial distance in the outer region (measured up to $R \approx 60^{\prime \prime}$ ). The profiles in Fig. 6 are obtained from a computation with primordial binaries in presence of a solar-neighborhood-like external Galactic potential.

The above values of the parameters $\epsilon, \tau_{d}$ and $\tau_{g}$, quantifying the overall gas expulsion, fair well with several young clusters (see above) and hence can be considered well-representative. However, it would be of interest to consider how our conclusions can be affected by moderate alterations of these parameters, representing possible cluster-to-cluster variations. As shown in Banerjee \& Kroupa (2013), the effect of varying the delay time, $\tau_{d}$, is simply a time-shift in the cluster's violent expansion without substantially affecting the cluster's evolution for $t>\tau_{d}$. Hence, a delay of $\tau_{d}+\delta \tau_{d}$ in our computed models would reproduce HD97950 at $\approx t+\delta \tau_{d}$. As such, one
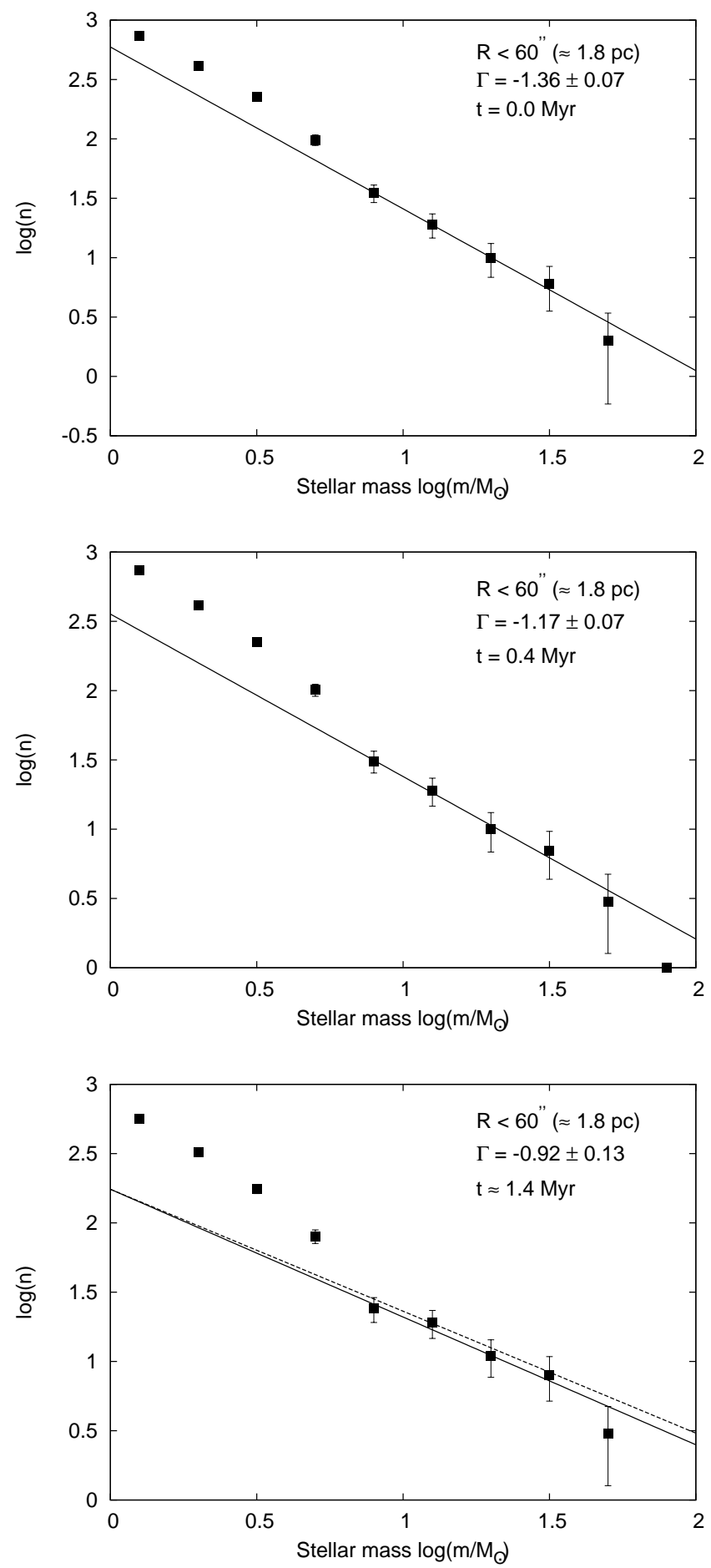

Figure 5. The stellar mass function (of primaries) within the central $60^{\prime \prime}(\approx 1.8 \mathrm{pc})$ of the computed model with primordial binaries (model HD97950b) at $t \approx 0.0,0.4$ and $1.4 \mathrm{Myr}$ (top, middle and bottom panels respectively). Beginning with the global canonical value of $\Gamma=-1.35\left(\alpha_{2}=2.35\right)$, the MF slope develops a break with time as a result of the switching to much tighter initial orbital periods for massive binaries (from $>5 M_{\odot}$; see Sec. 3.1). Upwards the break $\left(\right.$ at $\left.\log \left(m / M_{\odot}\right) \approx 0.7\right)$, the MF slope becomes increasingly shallow while it remains close to canonical for lower $m$. The corresponding slope at $t \approx 1.4 \mathrm{Myr}$ (bottom), viz., $\Gamma=-0.92 \pm 0.13$ (solid line), agrees closely with the corresponding measured one, viz., $\Gamma=-0.88 \pm 0.15$ (dashed line; Pang et al. 2013). 

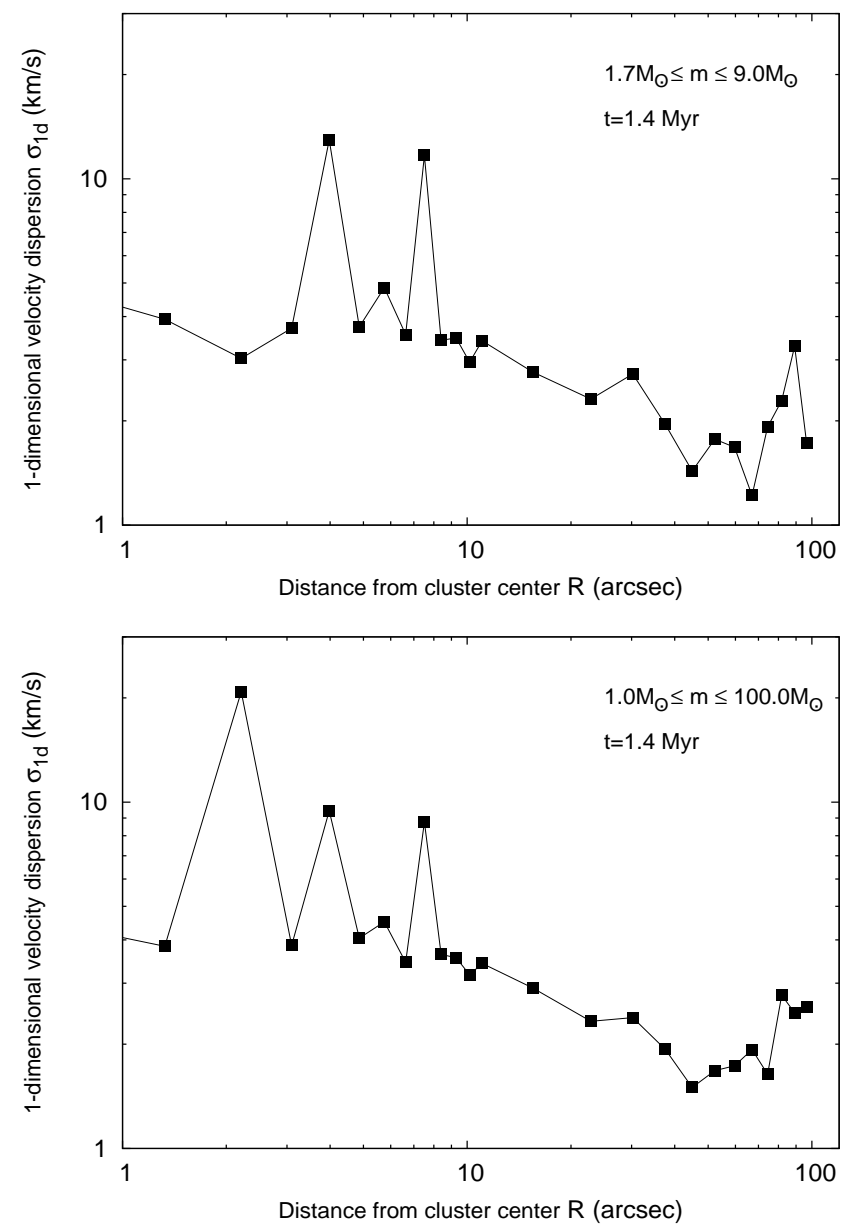

Figure 6. Radial variation of 1-dimensional velocity dispersion, $\sigma_{1 \mathrm{~d}}$, for the computed model with primordial binaries (in presence of a tidal field) at $t=1.4 \mathrm{Myr}$ for stellar mass ranges $1.7 M_{\odot} \leq m<$ $9.0 M_{\odot}($ top panel $)$ and $1.0 M_{\odot} \leq m \leq 100.0 M_{\odot}($ bottom panel $)$. The overall increasing trend of $\sigma_{1 \mathrm{~d}}$ with $R$ in the outer regions $\left(R \gtrsim 40^{\prime \prime}\right.$ in this case) indicates a recent gas expulsion. This can be tested in future by more accurate proper motion measurements (e.g., by Gaia) in the outer regions of the HD97950 cluster. The tangential velocities of selected stars in Pang et al. (2013) (for $\left.R \lesssim 60^{\prime \prime}\right)$ do show an increasing trend with $R$.

can allow for $\delta \tau_{d} \approx \pm 0.1 \mathrm{Myr}$ in the models computed here to obtain similarly good matchings.

For $\epsilon<50 \%$, as in the present case, the bound fraction of stars that re-virializes to form the remnant cluster (Kroupa et al. 2001; Banerjee \& Kroupa 2013) depends sensitively on $\tau_{g}$ (Baumgardt \& Kroupa 2007). However, the computed clusters here, which are still mostly expanding at the HD97950's age of $t \approx 1 \mathrm{Myr}$, are far from re-gaining dynamical equilibrium (see above; it takes $\approx 3$ Myr to re-virialize an HD97950-like cluster as shown in Banerjee \& Kroupa 2013) but still retains a compact spatial distribution. Therefore, at $t \approx 1 \mathrm{Myr}$, most of the cluster's primordial stellar population contributes to the computed radial profiles and the other quantities. Hence, the model-observation agreements, as presented here, would still mostly persist with a moderate change of $\tau_{g}\left(v_{g}\right)$ as long as the gas-removal is explosive (see Sec. 2.1.1). The computed values of $\sigma_{1 \mathrm{~d}}$ can be expected to increase moderately with decreasing $\tau_{g}$ but this trend is likely to be suppressed by the substantial fluctuations in the values of $\sigma_{1 \mathrm{~d}}$ (see Sec. 3, Fig. 3).

With a substantially higher $\epsilon$, one would expect a $M_{\text {ecl }}(0) \approx 20000 M_{\odot}$ cluster to be even more overdense/oversized and a matching model would be of $M_{\text {ecl }}(0) \lesssim 10000 M_{\odot}$, i.e., below HD97950's photometric mass limit. The reproducibility of the NGC3603's young cluster as a function of the parameters $\epsilon, \tau_{d}$ and $\tau_{g}$ will be investigated in detail in a future project. An immediate improvement over this work would be to apply colour filters (e.g., V and I) to the stellar luminosities to calculate the density profiles and the mass functions for comparisons with observations even more accurately.

We thank Andera Stolte of the Argelander-Institut für Astronomie, Bonn and Wolfgang Brandner of the MaxPlanck-Institut für Astronomie, Heidelberg for motivating discussions. We thank the anonymous referee for constructive suggestions leading to substantial improvement of the presentation and the discussions.

\section{REFERENCES}

Aarseth, S.J. 2003, "Gravitational N-Body Simulations".

Cambridge University Press.

Adams, F.C. 2000, ApJ, 542, 964.

Anderson, L.D., Bania, T.M., Jackson, J.M., et al. 2009, ApJS, 181,255

André, P., Meńshchikov, A., Koenyves, V., et al. 2011, in Alfaro

Navarro, E.J., Gallego Calvente, A.T., Zapatero Osorio, M.R. (Eds.) Stellar Clusters \& Associations: A RIA Workshop on Gaia. Granada, Spain: IAA-CSIC, 321.

Banerjee, S. and Kroupa, P. 2013, ApJ, 764, 29.

Bate M.R. and Bonnell, I.A. 2004, in Lamers, H.J.G.L.M., Smith, L.J., Nota A. (Eds.) The Formation and Evolution of Massive Young Star Clusters, (ASP Conf. Proc. 322). San Francisco: Astronomical Society of the Pacific, 289.

Bate M.R., 2009, MNRAS, 392, 590.

Bate, M.R. 2012, MNRAS, 419, 3115

Bate, M.R., Tricco, T.S., Price, D.J. 2013, MNRAS(doi:10.1093/mnras/stt1865)

Baumgardt, H. and Kroupa, P., 2007, MNRAS, 380, 1589.

Boily, C. and Kroupa, P. 2002, in Grebel E.K., Brandner, W. (Eds.) Modes of Star Formation and the Origin of Field Populations, (ASP Conf. Proc. 285). San Francisco: Astronomical Society of the Pacific, 141.

Chini, R., Hoffmeister, V.H., Nasseri, A., et al. 2012, MNRAS, $424,1925$.

Churchwell, E., 2002, ARA\&A, 40, 27.

Fujii, M.S., Saitoh, T.R. and Portegies Zwart, S.F. 2012, ApJ, $753,85$.

Geyer, M.P. and Burkert, A. 2001, MNRAS, 323, 988.

Girichidis, P., Federrath, C., Banerjee, R. and Klessen, R.S. 2011 MNRAS, 413, 2741.

Girichidis, P., Federrath, C., Allison, R., et al. 2012, MNRAS, $420,3264$.

Harayama, Y., Eisenhauer, F. and Martins, F. 2008, ApJ, 675, 1319.

Heggie, D.C. and Hut, P. 2003, "The Gravitational Millon-Body Problem: A Multidisciplinary Approach to Star Cluster Dynamics". Cambridge University Press, Cambridge, UK.

Hennemann, M., Motte, F., Schneider, N., et al. 2012, A\&A, 543, L3.

Hills, J.G. 1980, ApJ, 235, 986.

Hill, T., Motte, F., Didelon, P., et al. 2011 A\&A, 533, A94.

Hurley, J.R., Pols, O.R. and Tout, C.A. 2000, MNRAS, 315, 543.

Klessen, R.S., Burkert, A. and Bate, M.R. 1998, ApJ, 501, L205.

Kroupa, P. 1995a, MNRAS, 277, 1491.

Kroupa, P. 1995b, MNRAS, 277, 1507.

Kroupa, P., Petr, M.G. and McCaughrean M.J. 1999, NewA, 4, 495.

Kroupa, P. 2001, MNRAS, 322, 231

Kroupa, P. Aarseth, S. and Hurley, J. 2001, MNRAS, 321, 699

Kroupa, P. 2005, in Turon, C., O'Flaherty, K.S. and Perryman, M.A.C. (Eds.) The Three-Dimensional Universe with Gaia (ESA SP-576). 629p.

Kroupa, P. 2008, in Aarseth, S.J., Mardling, R.A. and Tout, C.A. (Eds.) The Cambridge N-body Lectures (Lect. Notes Phys. 760). Springer-Verlag Berlin Heidelberg. 
Kroupa, P., Weidner, C., Pflamm-Altenburg, J., et al. 2013, in Oswalt, T.D. and Gilmore, G. (Eds.) Galactic Structure and Stellar Populations (Planets, Stars and Stellar Systems, Volume 5). Springer Science+Business Media Dordrecht (2013). $115 p$.

Krumholz, M.R. and Matzner, C.D. 2009, ApJ, 703, 1352.

Küpper, A.H.W., Maschberger, T., Baumgardt, H. and Kroupa, P. 2011, MNRAS, 417, 2300.

Lada, C.J. and Lada, E.A. 2003, ARA\&A, 41, 57.

Longmore, S.N., Kruijssen, J.M.D., Bastian, N., et al. 2014, in Beuther, H., Klessen, R., Dullemond, C. and Henning, Th. (Eds.) Protostars and Planets VI, University of Arizona Press, preprint (arXiv:1401.4175).

Machida, M.N. and Matsumoto, T. 2012, MNRAS, 421, 588

Malinen, J., Juvela, M., Rawlings, M.G., et al. 2012, A\&A, 544, A50.

Marks, M. and Kroupa, P. 2011, MNRAS, 417, 1702.

Marks, M. and Kroupa, P. 2012, A\&A, 543, A8.

Moeckel, N., Holland, C., Clarke, C.J. and Bonnell, I.A. 2012, MNRAS, 425, 450.

Osterbrock, D.E. 1965, ApJ, 142, 1423

Pang, X., Grebel, E.K., Allison, R., et al. 2013, ApJ, 764, 73.

Patel, N.A., Curiel, S., Sridharan, T.K., et al., 2005, Nature, 437, 109.
Pfalzner, S. and Kaczmarek, T. 2013, A\&A, 555, A135.

Portegies Zwart, S.F., McMillan, S.L.W. and Gieles, M. 2010, ARA\&A, 48, 431.

Price, D.J. and Bate, M.R. 2010, in Plasmas in the laboratory and the Universe:Interactions, Patterns, and Turbulence, (AIP Conf. Proc. 1242), 205.

Rochau, B., Brandner, W., Stolte, A., Gennaro, M., et al. 2010, ApJ, 716, L90.

Sana, H. and Evans, C.J. 2011, in Neiner, C., Wade, G., Meynet, G. and Peters, G. (Eds.) Active OB Stars: Structure, Evolution, Mass Loss, and Critical Limits (IAU Symp. 272). Cambridge Univ. Press, Cambridge, 474.

Schneider, N., Csengeri, T., Bontemps, S., et al. 2010, A\&A, 520, A49.

Schneider, N., Csengeri, T., Hennemann, M., et al. 2012, A\&A, 540, L11.

Smith, R., Goodwin, S., Fellhauer, M. and Assmann, P. 2013, MNRAS, 428, 1303.

Stolte, A., Brandner, W., Brandl, B., et al. 2004, AJ, 128, 765.

Stolte, A., Brandner, W., Brandl, B., et al. 2006, AJ, 132, 253. 